(Aus der physiol. Abteilung des Instituts für exper. Medizin zu St. Petersburg.)

\title{
Zur Physiologie der Pankreassekretion.
}

Von

\section{A. J. Smirnow.}

Die Frage über die Wirkung des Fettes auf die Pankreassaftabsonderung hat bisher keinen völligen Abschluss gefunden. Die selbständige Wirkung des Fettes als Erregers der Pankreassekretion [Damaskin 1$)]$ wurde in Zweifel gezogen und die Vermutung ausgesprochen, dass das eigentliche Erregungsmoment beim Fett lediglich in den Fettsäuren zu suchen sei [Popielski²), Pflüger $\left.\left.{ }^{3}\right)\right]$. Da neutrales Fett unter dem Einfluss der fettspaltenden Fermente des Magen-, Darm- und vornehmlich des Pankreassaftes in seine Komponenten: Glycerin und Fettsäure zerfällt, wobei diese letztere dank den sich in das Lumen des Zwölffingerdarms ergiessenden alkalisch reagierenden Säften sich rasch in Seifen verwandelt, da könnte man meinen, dass safttreibend auf die Bauchspeicheldrüse nicht neutrales Fett an sich, vielmehr die Produkte seiner Zersetzung - Seifen und Fettsäuren - einwirken. Die safttreibende Wirkung dieser Substanzen (Glycerin erwies sich als indifferent) auf die Bauchspeicheldrüse wurde dargetan [Babkin ${ }^{4}$ ), Sawitsch ${ }^{5}$ ), Studsinski ${ }^{6}$ ), Bylina ${ }^{7}$ )];

1) N. J. Damaskin, Über die Wirkung des Fettes auf die Pankreassekretion. Verhandl. d. Gesellsch. russ. Ärzte zu St. Petersburg. 1895/1896. Februar. S. 7.

2) L. B. P p pielski, Über die sekretionshemmenden Nerven des Pankreas. Dissert. S. Petersburg. 1896.

3) E. Pflüger, Über die Verseifung, welche durch die Galle vermittelt wird, und die Bestimmung ron Seifen neben Fettsäuren in Gallenmischungen. Pflüger's Arch. Bd. 90 S. 1.

4) B. P. Babkin, L'influence des savons sur la sécrétion du pancréas. Arch. des Sciences Biologiques t. 11. 1904.

5) W. W. Sawitsch, Beiträge zur Physiologie der Pankreassaftsekretion. Zentralbl. f. d. ges. Physiol und Path. des Stoffwechsels 1909 Nr. 1.

6) J. B. Studsinski, Über den Einfluss der Fette und Seifen auf die sekretorische Tätígkeit des Pankreas. Russky Wratsch. 1911 Nr. 1, 2 und 3.

7) A. S. By lina, Normale Penkreassekretion als Synthese von nervösen und humoralem Einfluss. Pflüger's Arch. Bd. 142 S. 31. 
nichtsdesto weniger blieb jedoch die Frage über neutrales Fett als selbs tändigen Erreger der Pankreassekretion immer noch ungelöst.

Frühere Experimentatoren fübrten das Fett in den Magen ein, doch schloss diese Versuchsanordnung die Möglichkeit nicht aus, dass sich dem Fett Magensaftsäure beimengte. Wenn auch einerseits das in den Magen eingeführte Fett die Magensaftsekretion zum Stillstand bringt, so führt doch andrerseits der Übertritt eines Gemenges von Galle, Pankreas- und Darmsaft in den Magen zur Bildung von Seifen, die als energische spezifische Erreger der Magensaftabsonderung erscheinen (Piontkowsky'), Seljony und Sawits $\left.\mathrm{ch}^{2}\right)$ ]. Um eine solche Bedingung, die die Vornahme der Versuche in hohem Maasse beeinträchtigt, auszuschliessen, musste unser Versuch in der Weise angeordnet werden, dass der Magen vom Duodenum gleichsam abgetrennt bliebe und das Fett unmittelbar mit der Duodenalschleimhaut in Berührung käme.

Auf Anraten des hochverehrten Herru Prof. J. P. Pawlow stellte ich eine Reihe von Untersuchungen an hinsichtlich der Wirkung neutralen Fettes auf die Pankreassekretion unter unmittelbarer Reizung der Schleimhaut des Duodenums. Die Aufgabe der Untersuchung bestand darin: 1. die Möglichkeit auszuschliessen, dass irgendein anderes Reizmittel ausser Fett auf die Duodenalschleimhaut einwirke, 2. den Charakter der Pankreassekretion unter Einwirkung von Fett in qualitativer und qantitativer Beziehung festzustellen, und 3. die qualitative und quantitative Stite der Pankreasabsonderung bei Einführung von neutralem Fett in das Duodenum vor und nach subkutaner Injektion von $0,005 \mathrm{~g}$ Atropin gegenüberzustellen. Der Versuch wurde an einem Hunde vorgenommen, dem drei Fisteln angelegt waren: eine am Pankreas, eine am Magen und eine dritte am Darm im Bereich des Duodenums. Die beiden ersten Operationen waren bereits im Dezember 1910 ausgefülurt worden; die Anlegung der Darmfistel erfolgte im April 1911. Der Gesundheitszustand des Hundes ist während der ganzen Zeit ein vollkommen normaler, und das Tier macht den Eindruck eines durchaus lebensfrohen Indiviluums. Dieses Hundes bediente sich übrigens auch By lin a bei seinen Versuchen.

1) L. F. Piontkowski, Über die Wirkung der Seifen auf die Arbeit der Pepsindrüsen. Dissert. St. Petersburg. 1906.

2) G. P. Seljony und W. W. Sawitsch, Zur Physiologie des Pylorus. Verhanul. d. Gesellsch. russ. Ärzte zu St. Petersburg S. 221. 1910-1911. 
Im Verlauf der drei Monate, während welcher diese Arbeit vorgenommen wurde, liess das Tier nicht nur eine Gewichtsabnahme vermissen, nahm vielmehr um sechs Pfund an Körpergewicht $\mathrm{zu}$. Erkrankungen irgendwelcher Art sowie Durchfallerscheinungen, wie sie so oft bei Versuchen mit Fett beobachtet wurden, blieben aus; der Verdauungsapparat befand sich die ganze Zeit über in ausgezeichnetem Zustande. Die übliche Methodik der Verdauugsversuche erfubr einige Veränderungen infolge von Vorrichtungen, wie sie die spezielle Aufgabe im vorliegenden Fall erheischte. Wie stets, wurde bei geöffneter Duodenalfistel der Magen ausgespült, die Magenfistel während des ganzen Verlaufs des Versuchs offen gehalten und unter diese ein Gefäss zum Auffangen der abfliessenden Flüssigkeit gestellt. Die Darmfistel wurde mit einem Pfropfen geschlossen, durch den eine t-förmig gebogene Glasröhre hindurchgeführt war; der Inhalt dieser Röhre wurde während der ganzen Dauer des Versuches kontrolliert. Der Abflussarm der t-förmigen Röhre, die mit einer Bürette von $50 \mathrm{ccm}$ Inhalt in Verbindung stand, war mittelst einer Mohr'schen Klemme abgebunden und diente zur Entnahme des Zwölffingerdarminhalts behufs Untersuchung. Der Abfluss der Flüssigkeit aus der Bürette wurde durch eine Schraubenklemme reguliert. An der Bauchwand, der Pankreasfistel entsprechend, war ein Glastrichter befestigt, mittelst dessen der abfliessende Pankreassaft gesammelt wurde. Die Reaktion des Inhalts von Magen und Iluodenum wurde im Laufe des Experiments zu wiederholten Malen untersucht. Wo es sich um Untersuchung des Pankreassaftes auf Fermente handelte, wurden die gesammelten Saftportionen auf Eis gestellt. Die Bestimmung des Stickstoffgehaltes nach der Methode von $\mathrm{Kjeldahl} \mathrm{wurde} \mathrm{nach} \mathrm{jedem} \mathrm{einzelnen} \mathrm{der} \mathrm{Versuche} \mathrm{vorgenommen;}$ dasselbe geschal mit der Bestimmung des festen Rückstandes.

Sobald man auf Grund der Kontrollversuche mit $300 \mathrm{ccm}$ Milch als fetthaltiger Substanz mit Sicherheit annehmen konnte, dass die weiteren Versuche nicht an einem kranken Tiere vorgenommen würden, begannen die Untersuchungen hinsichtlich der Wirkung des Fettes auf die Schleimhaut des Duodenums. Ein so wertvoller Hund, wie er uns zur Verfügung stand, gab die Möglichkeit, den Versuch in der Weise anzuordnen, dass der Magen gleichsam physiologisch vom Duodenum abgetrennt blieb. Dies war eine der wichtigsten Versuchsbedingungen, da es nur bei solcher Versuchsanordnung möglich war, in das Duodenum verschiedene Substanzen 
einzuführen, ohne befürchten zu müssen, dass sich ihnen saurer Magensaft beimenge. Diese Abtrennung des Magens vom Duodenum wurde dadurch erreicht, dass man während der gesamten Beobachtungsdauer die Magenfistel offen hielt. Dass der ganze Mageninhalt wirklich nach aussen hin abfloss, ist aus der weiter unten angeführten Tabelle zu ersehen, wo zur Zeit des höchsten Stadiums der Magensaftsekretion die Kontrollbeobachtung hinsichtlich der Absoudernng des Pankreassaftes von 30 Minuten bis zu 1 Stunde 15 Minuten ganz unbedeutende Ziffern ergab.

Besonderes Interesse verdient in dieser Hinsicht der Versuch, bei welchem durch das Vorhandensein eines Stückes Fleisch im Magen aus diesem durch die geöffnete Fistel eine verbältnismässig grosse Saftmenge herausfloss und nichtsdestoweniger die Kontrollahsonderung in 1 Stunde 15 Minuten 1 ccm Pankreassaft mit beträchtlichem Stickstoffgehait $(0,8253)$ ergibt.

Kontrollbeobachtung vor Inangriffnahme eines jeden einzelnen Versuches mit Fett.

\begin{tabular}{|c|c|c|c|}
\hline Datum & Saftmenge & Reaktion im Magen & Beobachtungszeit \\
\hline $\begin{array}{l}\text { 7. Juli } \\
\text { 11. " } \\
\text { 13. " } \\
\text { 25. " } \\
\text { 10. August } \\
\text { 18. " " } \\
\text { 22. " }\end{array}$ & $\begin{array}{c}0,4 \\
0,4 \\
0,6 \\
1,0 \\
0,8 \\
2 \text { Tropfen } \\
1,0\end{array}$ & $\begin{array}{c}\text { neutral } \\
\text { neutral } \\
\text { schwach sauer } \\
\text { stark sauer } \\
\text { (im Magen Fleisch) } \\
\text { sauer } \\
\text { schwach sauer } \\
\text { schwach sauer }\end{array}$ & $\begin{array}{c}45^{\prime} \\
1 \mathrm{~h} \\
45^{\prime} \\
1 \mathrm{~h} 15^{\prime}(\mathrm{N}-0,8253) \\
45^{\prime}(\text { fester Rückstand } 6,4) \\
30^{\prime} \\
30^{\prime}(\mathrm{N}-1 \mathrm{~g})\end{array}$ \\
\hline
\end{tabular}

Um etwaige dahingehende Bedenken, diese unbedeutende Absonderung bei den Kontrollversuchen hinge von der aus dem Magen in das Duodenum übertretenden Magensaftsäure $a b$, zu entkräften, wurden von uns zwei Versuche mit unmittelbarer Einführung von $0,1 \% \mathrm{HCl}$ in das Duodenum vorgenommen, da eine solehe Lösung $\mathrm{HCl}$ als normaler Erreger der Pankreassekretion (unter direkter Einwirkung auf die Duodenalschleimbaut - Kat z n el s o n) anzusehen ist. Vergleicht man diese Daten mit der Kontrollabsonderung, so sieht man, dass die Saftsekretion auf Einführung einer $0,1 \%$ igen Lösung $\mathrm{HCl}$ in das Duodenum relativ gross ist und sich durch einen weit geringeren Fermentgehalt auszeichnet. 

I.
In 30 Min. . . 4,2 ccm
N . . . 0,4648
Fester Rückstand 3,5
II. $\quad 30 \quad, \quad \ldots 3,4 \quad$,
$\mathrm{N} \ldots \ldots, 0,4200$
Fester Rückstand 3,5

Diese Daten geben die Möglichkeit, die Frage dahin zu formulieren, dass, mag die unbedeutende Absonderung des Kontrollsafts auch von der Magensaftsäure abhängen, sie jedoch immerhin aus dem Magen in das Duodenum in einer so geringfügigen Quantität eindringt und einen so geringfügigen Prozentsatz an $\mathrm{HCl}$ enthält, dass sie bei den weiteren Versuchen mit Fett die Untersuchung nicht beeinträchtigen kann. Dies bestätigen die folgenden Versuche mit Fett, wo ein sehr auffallender Unterschied im Vergleich mit der Kontrollsekretion bemerkbar ist. Möglich, dass die Kontrullabsonderung des Pankreassaftes von Nerveneinflussen oder bisweilen von periodischen Zurückwerfungen (B oldyrew) abhängt, da solche Säfte einen hohen Stickstoffgehalt aufweisen.

Um eine Gewähr dafür zu haben, dass in das Duodenum zusammen mit dem Fett nicht auch Fettsäuren, wie sie in dem käuflichen Öl stets enthalten sind, mit übertreten, wurde das Öl neutralisiert; bei zwei Versuchen geschah dies mit Eiweiss (90 cem Öl + $10 \mathrm{ccm}$ Eiweiss), bei den übrigen Versuchen wurde zur Neutralisation heisses $\mathrm{Ba}(\mathrm{OH})_{2}$ verwendet. Die Neutralität des Fettes wurde nach der Methode von Burstyn bestimmt.

Die geöffnete Magenfistel hatte nicht nur den Zweck, den Magensaft abfliessen zu lassen, sie diente ferner auch zur Ableitung der Produkte der Fettverwandlung. Dieser letztere Umstand hatte eine grosse Bedeutung, da die im Dünndarm angesammelten Produkte der Fettspaltung die Periode der Pankreassaftabsonderung ausdehnen und beim Übertritt in den Magen bei geschlossener Magenfistel eine Sekretion des Magensaftes hervorrufen sowie zusammen mit diesem in den Dünndarm zurückgelangen konnten.

Neutrales Fett wurde in das Duodenum in geringen Portionen in Zeitabständen von je 5 Minuten eingeführt.

Bei einigen Versuchen wurden $50 \mathrm{ccm}$ Fett eingeführt; in der Mehrzahl der Fälle jedoch überstieg die eingeführte Fettmenge nicht $20-25 \mathrm{ccm}$. Die Einführung wurde in der Weise reguliert, dass das Fett spätestens 25 Minuten nach Beginn der Einführung in das Duodenum gelangte. 
7. Juli. Einführung einer Mischung von Fett mit Eiweiss. (Verhältnis: $90 \mathrm{ccm}$ Fett $+10 \mathrm{ccm}$ Eiweiss, eingeführt $50 \mathrm{ccm}$ der Mischung.) Magen ausgespült. Reaktion im Magen schwach sauer, vor Fetteinführung neutral.

\begin{tabular}{|c|c|c|c|c|}
\hline \multirow{2}{*}{ Zeit } & \multirow{2}{*}{$\begin{array}{c}\text { Saft- } \\
\text { menge } \\
\mathrm{ccm}\end{array}$} & \multicolumn{2}{|c|}{ Reaktion } & \multirow{2}{*}{ Bemerkungen } \\
\hline & & im Magen & im Duodenum & \\
\hline \multirow{3}{*}{ 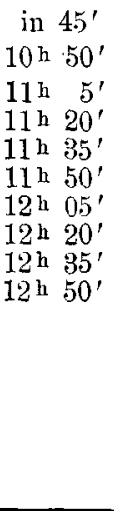 } & 0,4 & neutral & schwach sauer & vor Einfülı ung \\
\hline & Begil & der Fetteinf & ung in das Duo & enum (alle $\tilde{5}^{\prime} 10 \mathrm{ecm}$ ). \\
\hline & $\left.\left.\begin{array}{l}1,0 \\
1,8 \\
2,0 \\
1,2 \\
1,0 \\
0,9 \\
1,1 \\
1,0\end{array}\right\} \begin{array}{l} \\
\end{array}\right\}, 0$ & $\begin{array}{c}\text { alkalisch } \\
\text { sauer } \\
\text { sauer } \\
\text { sauer } \\
\text { sauer } \\
\text { sauer } \\
\text { sauer } \\
\text { sauer }\end{array}$ & $\begin{array}{c}\text { alkalisch } \\
\text { schwach alkal. } \\
\text { schwach alkal. } \\
\text { schwach alkal. } \\
\text { neutral } \\
\text { neutral } \\
\text { neutral } \\
\text { neutral }\end{array}$ & $\begin{array}{l}11^{\text {h }} 10^{\prime} \text {. Knurren im Darm; } \\
\text { aus dem Magen beginnt } \\
\text { emulgiertes Fett mit } \\
\text { Magensaft abgesondert zu } \\
\text { werden; bald wird bereits } \\
\text { eine seifenartige Fluissig- } \\
\text { keit von saurer Reaktion } \\
\text { abgesondert. Gleichzeitig } \\
\text { im t-förmigen Glasrohr } \\
\text { Färbung des Fettes durch } \\
\text { Galle. Fettfermente } 2,0 . \\
\text { Stickstoffmenge in } 100 \\
\text { ccm Saft } 0,7418 \text {. Fester } \\
\text { Rückstand in 100 cem } \\
\text { Saft } 5,2 \text {. }\end{array}$ \\
\hline Summa: & 10,0 & -- & 一 & - \\
\hline
\end{tabular}

10. August. Einführung neutralen Fettes $(25 \mathrm{ccm})$. Magen ausgespült. Vor Fetteinführung Reaktion im Magen sauer, im Duodenum schwach alkalisch (eher neutral).

\begin{tabular}{|c|c|c|c|c|}
\hline \multirow{2}{*}{ Zeit } & \multirow{2}{*}{$\begin{array}{c}\text { Saft- } \\
\text { menge } \\
\mathrm{ccm}\end{array}$} & \multicolumn{2}{|c|}{ Reaktion } & \multirow{2}{*}{ Bemerkungen } \\
\hline & & im Magen & im Duodenum & \\
\hline $\begin{array}{l}\text { in } 45^{\prime} \\
1^{\mathrm{h}} 00^{\prime}\end{array}$ & \multicolumn{4}{|c|}{$\begin{array}{c}\text { Beginn der Einführung neutralen Fettes in das Duodenum } \\
\text { (je } 5 \text { Portion von } 5 \mathrm{ccm})\end{array}$} \\
\hline $\begin{array}{ll}\text { 1h } & 15^{\prime} \\
1 \text { h } & 30^{\prime} \\
\text { 1 h } & 45^{\prime} \\
2 \text { h } & 00^{\prime} \\
2 \text { h } & 15^{\prime} \\
2 \text { h } & 30^{\prime} \\
2 \text { h } & 45^{\prime} \\
3 \text { h } & 00^{\prime}\end{array}$ & $\left.\begin{array}{l}1,0 \\
3,2 \\
3,4 \\
2,0\end{array}\right\} 9,6$ & $\begin{array}{c}\text { sauer } \\
\text { sauer } \\
\text { sauer } \\
\text { schwach sauer } \\
\text { schwach sauer } \\
\text { schwach sauer } \\
\text { sauer } \\
\text { sauer }\end{array}$ & $\begin{array}{l}\text { schwach alkal. } \\
\text { schwach alkal. } \\
\text { schwach alkal. } \\
\text { schwach alkal. } \\
\text { alkalisch } \\
\text { alkalisch } \\
\text { alkalisch } \\
\text { alkalisch }\end{array}$ & $\begin{array}{l}\text { h } 45^{\prime} \text { beginnt aus dem } \\
\text { Magen eine seifenartige } \\
\text { Flüssigkeit von schwach } \\
\text { saurer Reaktion abge- } \\
\text { sondert zu werden. In } \\
\text { dem t-förmigen Glasrohr } \\
\text { Fürbung um } 1 \text { h 25, } \\
\text { Menge des Stickstoffs in } \\
100 \text { cm Saft 0,5310. } \\
\text { Fester Rückstand in } \\
100 \text { ccm Saft } 4,0 \text {. Fester } \\
\text { Rückstand in 100 ccm } \\
\text { des vor Fetteinführung } \\
\text { gesammelten Saftes 6,4. }\end{array}$ \\
\hline $\mathrm{lmm}$ & 13,8 & 一 & 一 & - \\
\hline
\end{tabular}


13. August. Einführung neutralen Fettes $(25 \mathrm{ccm})$. Magen ausgespült. Vor Fetteinführung Reaktion im Magen schwach sauer, im Duodenum schwach sauer.

\begin{tabular}{|c|c|c|c|c|}
\hline \multirow{2}{*}{ Zeit } & \multirow{2}{*}{$\begin{array}{l}\text { Saft- } \\
\text { menge } \\
\text { ccm }\end{array}$} & \multicolumn{2}{|c|}{ Reaktion } & \multirow{2}{*}{ Bemerkungen } \\
\hline & & im Magen & im Duodenum & \\
\hline in $45^{\prime}$ & 0,6 & - & - & vor Fetteinführung \\
\hline $12^{\text {h } 30^{\prime}}$ & \multicolumn{4}{|c|}{$\begin{array}{l}\text { Beginn der Einführung neutralen Fettes in das Duodenum } \\
\left.\text { (je } 5^{\prime} \text { Portion von } 5 \mathrm{ccm}\right)\end{array}$} \\
\hline $\begin{aligned} 12 \text { h } & 45^{\prime} \\
1 \text { h } & 00 \\
1 \text { h } & 15 \\
1 \text { h } & 30 \\
1 \text { h } & 455^{\prime} \\
2 \text { h } & 00^{\prime}\end{aligned}$ & $\left.\begin{array}{l}\left.\begin{array}{l}2,6 \\
4,5 \\
2,2 \\
0,8\end{array}\right\} 10,1 \\
0,8 \\
0,6\end{array}\right\} 1,4$ & $\begin{array}{c}\text { schwach sauer } \\
\text { sauer } \\
\text { sauer } \\
- \\
\text { sauer } \\
\text { sauer }\end{array}$ & $\begin{array}{c}\text { neutral } \\
\text { neutral } \\
\text { neutral } \\
- \\
\text { neutral } \\
\text { neutral }\end{array}$ & $\begin{array}{l}\text { Im Laufe der sechsten } \\
\text { Minute in dem t-förmigen } \\
\text { Glasrohr Beginn der } \\
\text { Färbung. } 12 \mathrm{~h} 43^{\prime} \text { be- } \\
\text { ginnt aus dem Magen } \\
\text { eine seitenartige Flüssig- } \\
\text { keit von saurer Reaktion } \\
\text { abgesondert zu werden } \\
\text { (Knurren in den Därmen } \\
\text { und im Magen); der } \\
\text { Ausfluss dauert mit } \\
\text { Intervallen bis Al)lauf } \\
\text { der ersten Stunde. Stick- } \\
\text { stoffmenge in 100 ccm } \\
\text { Saft } 0,6200 \text {. Fester Rück- } \\
\text { stand in } 100 \mathrm{ccm} \text { Saft } 4,4 \text {. }\end{array}$ \\
\hline Summa: & 11,5 & - & - & $\ldots$ \\
\hline
\end{tabular}

11. Juli. Einführung einer Mischung von neutralem Fett und Eiweiss. Magen ausgespült. Vor Einführung der Mischung von neutralem Fett und Eiweiss in einer Quantität von $50 \mathrm{ccm}$, Reaktion im Magen neutral, im Duodenum schwach saner.

\begin{tabular}{|c|c|c|c|c|}
\hline \multirow{2}{*}{ Zeit } & \multirow{2}{*}{$\begin{array}{l}\text { Saft- } \\
\text { menge } \\
c \mathrm{~cm}\end{array}$} & \multicolumn{2}{|c|}{ Reaktion } & \multirow{2}{*}{ Bemerkungen } \\
\hline & & im Magen & im Duodenum & \\
\hline \multirow{3}{*}{$\begin{array}{c}10 \text { h } 30^{\prime} \\
\text { bis } 11 \text { b } 30^{\prime} \\
11 \text { h } 30^{\prime} \\
11 \text { h } 45^{\prime} \\
12 \text { h } 00^{\prime} \\
12 \text { h } 15^{\prime} \\
12 \text { h } 30^{\prime} \\
12 \text { h } 45^{\prime} \\
1 \text { h } 00^{\prime} \\
1 \text { h } 15^{\prime} \\
1 \text { h } 30^{\prime}\end{array}$} & \} 0,4 & - & - & vor Einführung \\
\hline & \multicolumn{4}{|c|}{ Beginn der Fetteinführung in das Duodenum (alle $5^{\prime} 10 \mathrm{ccm}$ ) } \\
\hline & $\begin{array}{l}\left.\begin{array}{l}0,2 \\
1,6 \\
3,6 \\
1,2\end{array}\right\} 6,6 \\
\left.\begin{array}{l}1,0 \\
0,4 \\
0,8 \\
0,6\end{array}\right\} 2,8\end{array}$ & $\begin{array}{c}-\overline{-} \\
\text { schwach saner } \\
\text { neutral } \\
\text { neutral } \\
\text { schwach alkal. } \\
\text { neutral } \\
\text { schwach sauer } \\
\text { sauer }\end{array}$ & \begin{tabular}{|c} 
neutral \\
neutral \\
neutral \\
neutral \\
neutral \\
neutral \\
neutral
\end{tabular} & $\begin{array}{l}11 \mathrm{~h} 55^{\prime} \text { sondert sich aus } \\
\text { dem Magen eine seifen- } \\
\text { artige Flüssigkeit von } \\
\text { schwach saurer Reaktion } \\
\text { ab. In dem t-törmigen } \\
\text { Glasrohr wird das Fett } \\
\text { durch Galle gefärbt. Im } \\
\text { Magen Knurren; abge- } \\
\text { sondert wird eine grosse } \\
\text { Menge seifenartiger Flüs- } \\
\text { sigkeit. Stickstoffgehalt } \\
\text { in } 100 \text { ccm Saft } 0,6420 . \\
\text { Fester Rückstand in } \\
100 \mathrm{ccm} \text { Saft } 4,75 \text {. }\end{array}$ \\
\hline Summa: & 9,4 & - & - & - \\
\hline
\end{tabular}


25. Juli. Einführung einer Mischung von neutralem Fett und Eiweiss (25 ccm). Im Magen ein kleines Stück Fleisch, dessen Entfernung nicht gelingt; aus der Fistel sondert sich Magensaft ab. Vor Fetteinführung Reaktion im Magen stark sauer, im Duodenum schwach sauer.

\begin{tabular}{|c|c|c|c|c|}
\hline \multirow{2}{*}{ Zeit } & \multirow{2}{*}{$\begin{array}{l}\text { Saft- } \\
\text { menge } \\
\text { ccm }\end{array}$} & \multicolumn{2}{|c|}{ Reaktion } & \multirow{2}{*}{ Bemerkungen } \\
\hline & & im Magen & im Duodenum & \\
\hline in $1^{\mathrm{h}} 15^{\prime}$ & 1,0 & stark sauer & schwach sauer & vor Einführung \\
\hline $10^{\mathrm{h}} 10^{\prime}$ & \multicolumn{4}{|c|}{$\begin{array}{l}\text { Beginn der Einführung einer Mischung aus neutralem Fett und Eiweiss } \\
\text { in das Duodenum (alle } 5^{\prime} 5 \mathrm{ccm} \text { ) }\end{array}$} \\
\hline 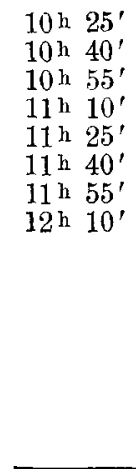 & $\left.\begin{array}{l}1,0 \\
3,4 \\
1,4 \\
2,0 \\
1,2 \\
1,4 \\
0,8 \\
0,8\end{array}\right\} 7,8$ & $\begin{array}{c}\text { sauer } \\
\text { sauer } \\
\text { sauer } \\
\text { saner } \\
\text { schwach sauer } \\
\text { schwach sauer } \\
\text { schwach sauer } \\
\text { saner }\end{array}$ & $\begin{array}{c}\text { neutral } \\
\text { neutral } \\
\text { neutral } \\
\text { schwach alkal. } \\
\text { alkalisch } \\
\text { alkalisch } \\
\text { alkalisch } \\
\text { alkalisch }\end{array}$ & $\begin{array}{l}10 \mathrm{~h} 35^{\prime} \text { in dem t-förmigen } \\
\text { Glasrohr Färbung durch } \\
\text { Galle. Gegen Ablauf einer } \\
\text { Stunde beginnt aus dem } \\
\text { Magen eine seifenförmige } \\
\text { Flüssigkeit abgesondert } \\
\text { zu werden; weiter nimmt } \\
\text { ihre Absonderung immer } \\
\text { zu. Menge des Stick- } \\
\text { stoffs in } 100 \text { cem Saft } \\
0,6383 \text { Stickstoffmenge } \\
\text { in } 100 \text { ccm des vor Fett- } \\
\text { einführung gesammelten } \\
\text { Saftes } 0,8253 \text {. Fester } \\
\text { Rückstand in } 100 \text { ccm } \\
\text { Saft } 4,55 \text {. }\end{array}$ \\
\hline Summa: & 12,0 & - & - & - \\
\hline
\end{tabular}

Alle von uns angestellten Versuche mit Fetteinführung in das Duodenum zeigen untereinander eine grosse Analogie. Aus den Versuchen ergibt sich, dass die Sekretion des Pankreassaftes in den ersten 15 Minuten nach Fetteinführung nicht bedeutend ist, wie dies bei allen Versuchen mit Fett der Fall zu sein pflegt, darauf gegen Ende der zweiten Viertelstunde stets zunimmt und sich auf einer gewissen Höhe hält, sodann jedoch abnimmt und allmählich bis zum Schluss des Versuches schwächer und schwächer wird. Der Eintritt einer bedeutenden Pankreassekretion ist immer von gewissen Erscheinungen begcleitet, die unfehlbar auf ein Höchststadium im Absonderungsprozess schliessen lassen. Anfänglich zeigt das Fett nicht die geringsten Veränderungen, wie man dies sehr bequem an dem mit der Darmfistel verbundenen t-förmig gebogenen Glasrohr beobachten kann. In dieser Zeit ist die Absonderung des Pankreassaftes nur unbedeutend. Gewöhnlich stellt sich 20-25 Minuten nach Beginn der Fetteinführung in den Därmen ein Knurren ein, und danach lassen sich in dem t-förmigen Glasrohr Gallestreifchen 
wahrnehmen. Jetzt nimmt die Pankreassekretion zu und erreicht mit der intensivsten Färbung der in dem t-förmigen Glasrohr enthaltenen Flüssigkeit ihren Höhepunkt. Fast gleichzeitig mit diesen Erscheinungen tritt aus der geöffneten Magenfistel emulgiertes Fett, das bald den Charakter einer seifenartigen Flüssigkeit annimmt, von gleicher Farbe und Zusammensetzung wie die Flüssigkeit in dem mit der Duodenalfistel verbundenen t-förmig gebogenen Glasrohr. Diese Flüssigkeit wird bis zum Schluss der ersten oder Anfang der zweiten Stunde abgesondert; zunächst ist ihre Reaktion infolge Vorhandenseins von Magensaft im Magen sauer, nimmt jedoch sodann unter gleichzeitiger Seifenbildung im Dünndarm neutrale oder alkalische Eigenschaften an. Sobald die Zurückwerfung dieser seifenartigen Flüssigkeit aufhört, lässt sich im Magen eine Abnahme der Pankreassekretion beobachten. Der Inhalt des Duodenums bleibt während der ganzen Versuchsdauer neutral oder alkalisch - ein Umstand, der darauf hinweist, dass, mögen dort auch immerhin Fettsäuren zur Bildung gelangen, diese keinen konstanten Charakter aufweisen und von alkalisch reagierenden Säften des Dünndarms rasch neutralisiert werden.

Aus den angeführten Versuchen ist ersichtlich, dass mit einer Steigerung der Pankreassekretion stets ein Frscheinen von Galle Hand in Hand geht. Im gegebenen Falle leuchtet ein, dass neutrales Fett als selbständiger Erreger eine nur schwache Wirkung auf die sekretorische Fähigkeit des Pankreas ausübt. Das Moment der Spaltung sowie. der darauf folgende Prozess der Zersetzung des Fettes erhöht dagegen die Absonderung des Pankreassaftes. Diese Erhöhung der Sekretion, die mit der wahrnehmbaren Verwandlung des Fettes zusammenfällt, kann auf die Funktionen zweierlei verschiedener Mechanismen zurückgeführt werden.

Im Augenblick der Spaltung können die sich bildenden Fettsäuren sowie ihre im Wege der Neutralisation der Fettsäuren durch neutrale und alkalisch reagierende Darmsäfte zur Entstehung gelangenden Salze wirksam sein. Die unbedeutende Absonderung des Pankreassaftes in der ersten Zeit nach Einführung des Fettes in das Duodenum kann gleichfalls auf die Spaltung zurückgeführt werden, die anfänglich langsam eintritt, später jedoch beträchtliche Dimensionen annimmt. Die oben angeführten Versuche schliessen die Möglichkeit einer solchen Wirkung der Fettsäuren im Augenblick der Spaltung keineswegs aus. Schliesslich kann man auch die An- 
nahme gelten lassen, dass Fett als solches, d. h. in unzersetzter Form, eine safttreibende Wirkung auf das Pankreas ausübt; diese Wirkung des Fettes ist schwach und wird im Augenblick seiner Spaltung erhöht (Erscheinen von Galle in dem t-förmigen Glasrohr).

Was den Fermentgehalt im erzielten Saft anbetrifft, so sind, wenn man den hohen Gehalt an Stickstoff und festem Rückstand [Babkin und Tichomirow $\left.\left.{ }^{1}\right)\right]$ in Betracht zieht, die Fermente in dem bei Fetteinführung in das Duodenum erlangten Saft ziemlich stark und können in keiner Weise als Hinweis darauf dienen, dass wir es hier mit einem Saft zu tun haben, der als Resultat der Einwirkung irgendeines anderen Reizmittels ausser Fett auf das Pankreas erzielt wird.

Der Gehalt an Stickstoff und festem Rückstand, wie er im Pankreassaft nach Fetteinführung in das Duodenum beobachtet wird (bei meinen Versuchen), ist fast identisch mit dem Gehalt an Stickstoff und festem Rückstand in dem nach Fetteinführung in den Magen erzielten Safte [Bylin $\left.\mathbf{a}^{2}\right)$ ].

Menge des Stickstoffs und festeh Rückstandes im Pankreassaft auf Fett.

Bei Einführung von $100 \mathrm{ccm}$ neutralen Fettes in den Magen [Bylina $\left.{ }^{2}\right)$.

\begin{tabular}{c|c}
\hline $\mathrm{N}$ & $\begin{array}{c}\text { Fester } \\
\text { Rückstand }\end{array}$ \\
\hline 0,5432 & 3,8 \\
0,5723 & 3,7 \\
0,6955 & 4,56 \\
0,6507 & 4,4 \\
0,6235 & 4,5 \\
0,6107 & 4,9
\end{tabular}

Bei Einführung von $50-20-25 \mathrm{ccm}$ neutralen Fettes in das Duodenum (Autor).

\begin{tabular}{c|c}
\hline \hline $\mathrm{N}$ & $\begin{array}{c}\text { Fester } \\
\text { Rückstand }\end{array}$ \\
\hline 0,5481 & 4,0 \\
0,7418 & 5,2 \\
0,6200 & 4,4 \\
0,6420 & 4,75 \\
0,6383 & 4,55 \\
0,6048 & 4,1
\end{tabular}

Auf Grund des angeführten Materials konnte man zur Erörterung darüber übergehen, welcher Art der Mechanismus sei, der bei der Wirkung des Fettes auf die Pankreassekretion im Falle unmittelbarer Fetteinführung in das Duodenum in Frage käme. Da

1) B. P. Babkin und N. P. Tichomirow, Zur Frage der gegenseitigen Beziehungen zwischen der proteolytischen Kraft, dem Stickstoffgehalt und dem Gehalt an festen Bestandteilen im Saft der Bauchspeicheldrüse. Hoppe-Seyler's Zeitschr. f. physiol. Chemie Bd. 62 S. 468.

2) Bylina, 1. c. 
nach der zurzeit herrschenden Ansicht die Tätigkeit der Bauchspeicheldrüse das summarische Resultat der Funktionen zweierlei verschiedener Mechanismen: des humoralen-chemischen sowie des nervösen, darstellt, so musste bei unseren Untersuchungen wünschenswert erscheinen der Aufklärung der Frage näher zu kommen, auf welchen Mechanismus die Pankreassekretion im Falle direkter Einführung von Fett in das Duodenum zurückzuführen sei. Die weiter unten angeführten Untersuchungen bilden einen der Versuche zur Aufklärung dieser komplizierten Frage. In den Fällen, wo es sich darum handelt, eine von diesen mit der Tätigkeit der Bauchspeicheldrüse verbundenen Funktionen - im vorliegenden Falle die nervöse auszuschalten, wendet man Atropin an. Dieses Alkaloid, das die nervöse Funktion paralysiert, gibt mehr oder weniqer die Möglichkeit auf die Wirkung der andern Funktion des Mechanismus, der

18. August. Einführung von neutralem Fett und subkutane Injektion von $0,005 \mathrm{~g}$ Atropin. Im Magen -.- Reaktion schwach sauer. Pulsfrequenz 80 in der Minute. Im Duodenum - Reaktion neutral. Vor Fetteinführung in $30^{\prime}-$ 2 Tropfen Pankreassaft. $12 \mathrm{~h} 45^{\prime}-$ Beginn der Einführung neutralem Fetts in das Duodenum im Betrage von $50 \mathrm{ccm}$.

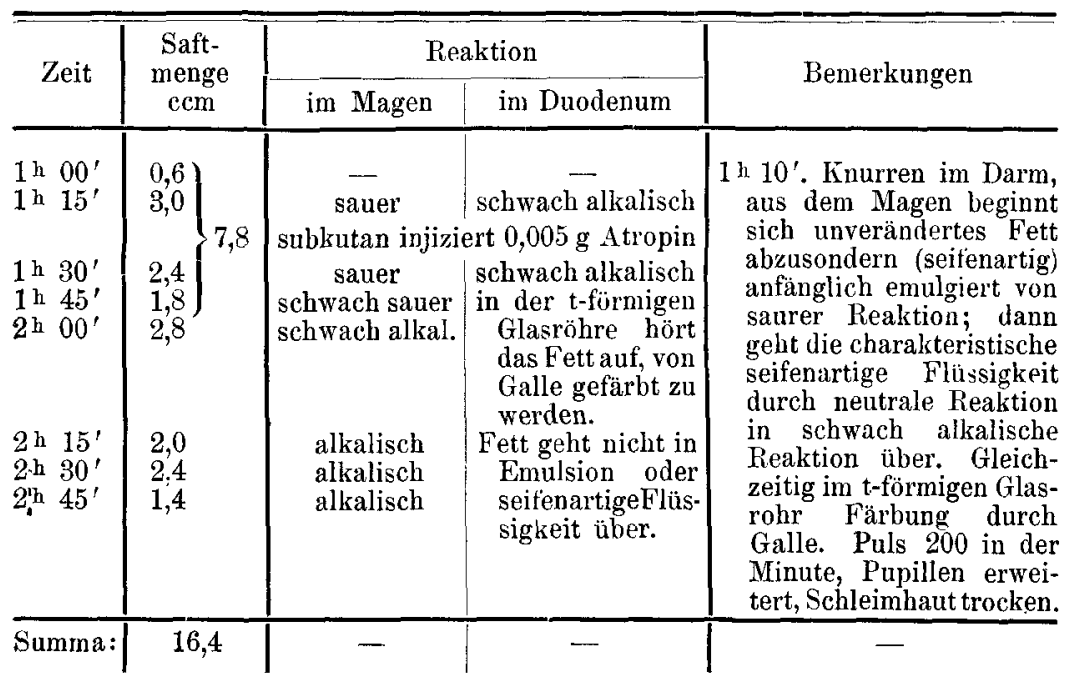

$N$ in $100 \mathrm{ccm}$ Saft: Fester Rückstand in $100 \mathrm{ccm}$ Saft:

\begin{tabular}{l|l} 
I. $30^{\prime}$ vor Atropininjektion $-0,8400 \mathrm{~g}$ & I.
\end{tabular}

II. $15^{\prime}$ nach " 0,2576, II.

III. $45^{\prime} " \quad " \quad 0,1570 "$ III. $45^{\prime}$ nach Atropininjektion $-1,7 \mathrm{~g}$,

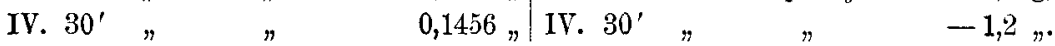


22. August. Einführung einer Mischung von neutralem Fett und Fiweiss sowie subkutane Injektion von Atropin. Im Magen - schwach saurer Schleim. l'ulsfrequenz 80 in der Minute. Im Duodenum - Reaktion neutral. Vor Einführung der Mischung in $30^{\prime} 10 \mathrm{ccm}$ Pankreassaft. $12 \mathrm{~h} 55^{\prime}$ Beginn der Einführung von einer Mischung neutralen Fettes und Eiweiss in das Duodenum im Betrage von $20 \mathrm{ccm}$ in Portionen von $4 \mathrm{ccm}$ pro $5^{\prime}$.

\begin{tabular}{|c|c|c|c|c|}
\hline \multirow{2}{*}{ Zeit } & \multirow{2}{*}{$\begin{array}{c}\text { Saft- } \\
\text { menge } \\
\text { ccm }\end{array}$} & \multicolumn{2}{|c|}{ Peaktion } & \multirow{2}{*}{ Bemerkungen } \\
\hline & & im Magen & im Duodenum & \\
\hline 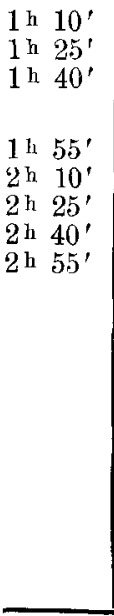 & $\left.\left.\begin{array}{l}0,4 \\
1,0 \\
3,4 \\
2,0\end{array}\right\} \begin{array}{l} \\
2,0 \\
1,6 \\
1,2 \\
1,0\end{array}\right\} 5,8$ & $\begin{array}{c}\text { - } \\
\text { - } \\
\text { subkutan injiziert } \\
\text { schwach alkalisch } \\
\text { neutral } \\
\text { schwach alkalisch } \\
\text { schwach alkalisch } \\
\text { schwach alkalisch }\end{array}$ & \begin{tabular}{|l}
$-\overline{-}$ \\
$0,005 \mathrm{~g}$ Atropin \\
neutral \\
neutral \\
neutral \\
neutral \\
neutral
\end{tabular} & $\begin{array}{l}1 \text { h } 20 \text { '. Knurren jm Daim, } \\
\text { gleichzeitig Beginn einer } \\
\text { energischen Pankreas- } \\
\text { saftabsonderung. In dem } \\
\text { t-förmigen Glasrohr Für- } \\
\text { bung durch Galle. Aus } \\
\text { dem Magen sondert sich } \\
\text { emulgiertes Fett ab. Im } \\
\text { Duodenum Reaktion neu- } \\
\text { tral. } 1 \text { - } 40^{\prime} \text { wird aus } \\
\text { dem Magen bereits eine } \\
\text { seifenartige Flüssigkeit } \\
\text { von schwach saurer Re- } \\
\text { aktion abgesondert; dann } \\
\text { geht die Reaktion allmäh- } \\
\text { lich in eine neutrale und } \\
\text { von einer solchen nach } \\
\text { und nach in eine schwach } \\
\text { alkalische über. Puls } 200 \\
\text { in der Minute. Vergif- } \\
\text { tungserscheinung. }\end{array}$ \\
\hline Summa: & 12,2 & - & - & - \\
\hline
\end{tabular}

$\mathrm{N}$ in $100 \mathrm{ccm}$ Saft.

Fester Rückstand in $100 \mathrm{ccm}$ Saft.

I. Vor Fetteinführung . . 1,000 g

II. $45^{\prime}$ vor Atropininjektion . $0,6049 \mathrm{~g}$

I.

III. $15^{\prime}$ nach

IV. $30^{\prime}$

$0,4256 \mathrm{~g}$

II. $45^{\prime}$ vor Atropininjektion . . 4,1 g

IV. $30^{\prime} "$ "

$0,1625 \mathrm{~g}$ IV. $30^{\prime}$

$0,1572 \mathrm{~g} \mid \mathrm{V} .30^{\prime}$ "

. . $2,9 \mathrm{~g}$

V. $30^{\prime} " \Rightarrow$

$n$

. $1,7 \mathrm{~g}$

chemischen, einen Schluss zu ziehen. Bei meinen Versuchen bediente auch ich mich dieses Giftes, um festzustellen, welche Wirkung schwefelsaures Atropin, in einer Quantität von $0,005 \&$ subkutan injiziert, auf die sekretorische Tätigkeit der Bauchspeicheldrüse ausübt. Die Anwendung einer solchen Dosis Atropin konnte dem Tier nicht nachträglich sein, musste aber auf den sensiblen nervössekretorischen Apparat der Drüse einen Einfluss ausüben. Die hier angeführten beiden Versuche zeigten ein gleiches Bild der Vergiftung, das sich in einer Steigerung der Pulsfrequenz von 80 bis zu 200 Sehlägen in der Minute, einer Erweiterung der Pupillen und einer 
allgemeinen Reflexerhöhung äusserte. Die Injektion von $0,005 \mathrm{~g}$ Atropin wurde vorgenommen, sobald bei dem in das Duodenum eingeführten Fett der übliche Spaltungstyp beobachtet wurde. In der Regel sank bereits 15 Minuten nach der Atropininjektion der Stickstoffgehalt bedeutend, und diese Abnahme des Stickstoffgehalts und festen Rückstandes (folglich auch der Fermente) währte bis Schluss des Versuchs. Die quantitative Seite der Pankreassekretion wurde jedoch von der Atropininjektion in Höhe von $0,005 \mathrm{~g}$ nicht in Mitleidenschaft gezogen; die Absonderung des Pankreassaftes erfuhr keine merkliche Veränderung.

Analoge Resultate erlangte Bylina, der Untersuchungen mit Fett anstellte, wobei er dieses in den Magen einführte und die Pankreassaftabsonderung nach subkutaner Injektion von $0,005 \mathrm{~g}$ Atropin beobachtete. Aus der angeführten Gegenüberstellung des Stickstoffgehalts und festen Rückstandes im Pankreassaft; der nach Injektion von $0,005 \mathrm{~g}$ Atropin im Höchststadium der Pankreassekretion erzielt wurde, ergibt sich die Identität seiner Ergebnisse mit den Resultaten meiner Versuche.

\begin{tabular}{|c|c|c|}
\hline \multirow{2}{*}{$\begin{array}{c}\text { Fett } \\
\text { in den Magen eingeführt } \\
\text { (Bylina) 18. April } \\
\text { vor Atropininjektion }\end{array}$} & \multicolumn{2}{|c|}{ Fett in das Duodenum eingeführt } \\
\hline & $\begin{array}{l}\text { (Autor) 18. August } \\
\text { vor Atropininjektion }\end{array}$ & $\begin{array}{l}\text { (Autor) 22. August } \\
\text { vor Atropininjektion }\end{array}$ \\
\hline 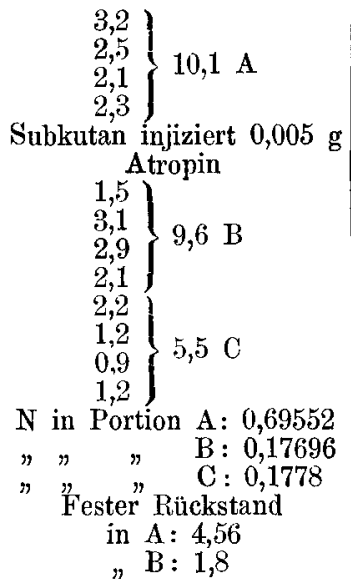 & 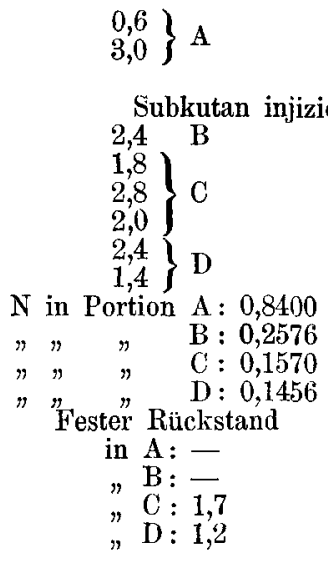 & 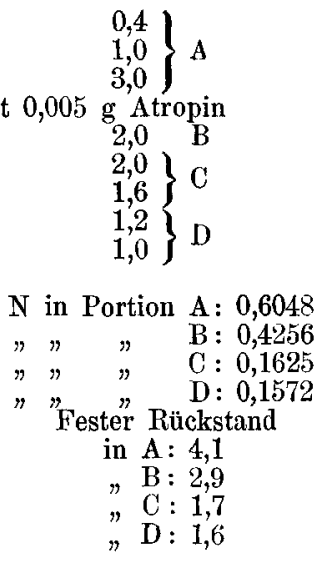 \\
\hline
\end{tabular}

Ein allmähliches Abfallen ist bei Bylina nicht bemerkbar, offenbar, weil es sich hier um eine Untersuchung der stündlichen Portionen des Pankreassaftes auf Stickstoff handelt, während bei uns 
die Bestimmung der Stickstoffmenge an ziemlieh häufigen Portionen vorgenommen wurde.

Zum Schluss möchte ich einen Versuch mit Atropin anführen, der in der Weise angeordnet war, dass zunächst eine subkutane Injektion von $0,005 \mathrm{~g}$ Atropin vorgenommen und erst hiernach neutrales Fett in das Duodenum eingeführt wurde. Da in dieser Form nur ein einziger Versuch angestellt wurde - eine Wiederholung misslang infolge ausserhalb des Versuches liegender Ursachen -, so muss es gewagt erscheinen, ihm irgendwelche Bedeutung beizumessen und sich zu Erklärungen verleiten zu lassen, die möglicherweise jeglicher Grundlage entbehren. Sollten die weiteren Untersuchungen dartun, dass im gegebenen Falle der Stillstand der Sekretion nicht durch zufällige Ursachen, wie etwa Erkrankung des Darmkanals, infolge deren die Spaltung des Fettes durch alkalisch rèagierende Säfte im Dünndarm zur Sistierung gelangte, hervorgerufen worden war, so kann diesem Versuch eine gewisse Bedeutung nicht abgesprochen werden.

Versuch mit subkntaner Atropininjektion vor Fetteinführung in das Duodenum. 3. August 1911.

Reaktion im Magen sauer.

Reaktion im Duodenum schwach alkalisch. Kontrollabsonderung im Verlaufe einer Stunde $-3 \mathrm{ccm}$.

1 h $10^{\prime}-0,005$ g Atropin subkutan injiziert.

$1^{\text {th }} 15^{\prime}$ - Beginn der Einführung einer Mischung von neutralem Fett und Eiweiss in Portionen von $5 \mathrm{ccm}$ für jede 5 Minuten bis zur Gesamtmenge von $25 \mathrm{ccm}$.

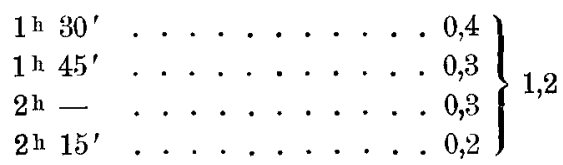

Während der achten Minute sind die Pupillen erweitert und die Pulsfrequenz bis zu 200 Schlägen in der Minute angestiegen; gegen Ende der Stunde zeigt der Hund eine grosse Unruhe und sucht sich aus dem Gestell loszureissen.

Nach einer einstündigen Versuchsdauer wurde das Fett abgelassen; es wies nicht die geringsten Veränderungen auf und enthielt keine Gallebestandteile. In dem $t$-förmig gebogenen Glasrohr hatte während der ganzen Zeit auch nicht die schwächste Färbung wahrgenommen werden können. Der Magen zeigt eine saure Reaktion, die gegen Ende der Stunde allmählich in eine schwach alkalische übergeht.

Stickstoffmenge in $100 \mathrm{ccm}$ Kontrollsaft . . . . . 0,840 g

Stickstoffmenge in $100 \mathrm{ccm}$ Saft nach Injektion von Atropin 0,560 , 
Fasst man die obigen Untersuchungsergebnisse zusammen, so kann man zu folgenden Schlussfolgerungen gelangen:

1. Die Absonderung des Pankreassaftes geht anfänglich im Verlauf von 20-25 Minuten nach Fetteinführung in das Duodenum offenbar bei neutraler oder alkalischer Reaktion des Duodenalinhalts vor sich.

2. Gleichzeitig mit der Fettspaltung nimmt die Pankreassekretion bedeutend zu.

3. Jer bei Einführung von neutralem Fett in das Duodenum zur Absonderung gelangende Pankreassaft zeichnet sich durch hohen Gehalt an Stickstoff und an festem Rückstand und folglich auch an Fermenten aus.

4. Eine subkutane Injektion von $0,005 \mathrm{~g}$ Atropin im Höchststadium der Fettspaltung verringert nicht die Quantität des zur Absonderung gelangenden Pankreassaftes, führt aber zu einer bedeutenden Verringerung des Gehalts an Stickstoff und festem Rückstand bereits nach Ablauf von 15 Minuten, wobei diese Verringerung auch weiter anhält. 